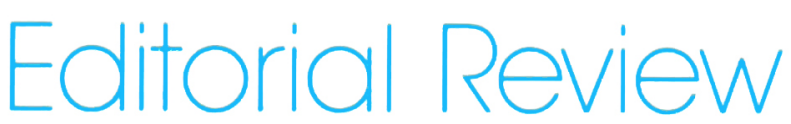

By F.D. Daschner, Prof, DrMed

\title{
The Transmission of Infections in Hospitals by Staff Carriers, Methods of Prevention and Control
}

Since the times of Semmelweis, life-threatening hospital-acquired infections have been transmitted on the hands of hospital personnel. For several years Semmelweis desperately tried to convince the colleagues in his gynecological department to wash and disinfect their hands. Most of the professors of surgery and gynecology in Vienna and other medical centers in Europe considered him a fool. They believed in the transmission of infectious diseases by some kind of miasma in the air. There has not been much change in the past 100 years. Hospital epidemiologists continue to teach that handwashing and hand disinfection is the most important measure in controlling cross-infection in hospitals. Many physicians still seem to believe that disinfection of air is as important as disinfection of hands. Other medical professionals sometimes consider hospital epidemiologists an ancient species who have learned nothing but to teach the gospel of Semmelweis.

Even today most hospital cross-infections are transmitted by hands, and hospital epidemiologists have to realize that the attitude of hospital personnel has not changed much since the time of Semmelweis.

Patients in intensive care units (ICU) are highly susceptible to nosocomial infections. It has therefore been suggested that ICU personnel wash their hands more frequently than personnel in other units (at least before and after contact with every patient). In 1981, 134 years after Semmelweis' original recommendation, Albert and Condie from the Washington Veterans Administration Center in Seattle evaluated the compliance of intensive care unit personnel with this internationally accepted recommendation. ${ }^{1}$ Contact with 28 patients was observed (40 different physicians and residents and 15 nurses had patient contact). Handwashing occurred after only $41 \%$ of the contacts. Physicians washed significantly less frequently than did nurses. For example, urine bags were manipulated, intravenous dressings were changed and respiratory equipment was adjusted without an intervening handwash. Only $28 \%$ of physicians in University hospitals and only $14 \%$ in private hospitals washed their hands after patient contact. Private patients-although paying more-might be at higher risk for acquiring nosocomial infections.

Larson investigated the hand flora of 103 hospital personnel over a mean of 35 days. One or more of 22 different species of gram-negative bacteria were found to be carried persistently on the hands of $21 \%$ of hospital personnel. Persons who washed hands less than 8 times per day were significantly more likely to carry the same species of gram-negative bacteria on the hands. Predominant organisms were species of Acinetobacter and Klebsiellal Enterobacter. ${ }^{2}$

Of 541 nosocomial infections over a 7 -month period, $21 \%$ were caused by species found on personnel hands. Again doctors washed hands significantly less often than nurses. Colonization of doctors' hands with gram-negative bacteria was significantly higher than that of nurses and other hospital personnel.

We investigated during a 7 -week period 328 hands of doctors and nurses in our medical intensive care unit. The results are summarized in the Table. Staphylococcus aureus was isolated from $20.5 \%$ of all hands. Twenty-seven percent of all hands were colonized with gram-negative bacteria. The organisms most commonly found were Enterobacter cloacae, Pseudomonas aeruginosa, Acinetobacter anitratus, Klebsiella pneumoniae and Enterobacter agglomerans.

It is interesting to note that certain gram-negative species such as Klebsiella and Enterobacter are more commonly isolated from hands than E. coli. Adams et al found that 3 of 11 subjects from whom Klebsiella pneumoniae was isolated carried this organism continuously over a period of 3 to 6 weeks; two of three carried Pseudomonas aeruginosa continuously and four of five of the control subjects carried the same biotype of Enterobacter agglomerans. ${ }^{3}$

Casewell and co-workers evaluated the survival of vari- 


\section{TABLE}

HAND CONTAMINATION OF PERSONNEL

IN A MEDICAL ICU (University Hospital,

Freiburg, 1983)

1. Doctors: $\quad 71,200 \mathrm{CFU} / \mathrm{Hand}$

Nurses: $\quad 39.800 \mathrm{CFU} / \mathrm{Hand}, \mathrm{P}<0.001$

2. $36 \%$ of all doctors' hands, $18 \%$ of all nurses' hands colonized with $S$. aureus, $P<0.005$

3. $21 \%$ of all doctors' hands, but only $5 \%$ of nurses' hands colonized with more than $1.000 \mathrm{CFU} / \mathrm{Hand}$ of $S$. aureus, $P<0.001$

4. $18 \% / 3 \%$ of doctors' hands, but $28 \% / 13 \%$ of nurses' hands colonized with gram-negative bacteria/Enterococci.

ous gram-negative bacilli; they found that three epidemic multiply-resistant strains of Klebsiella aerogenes survived better than Pseudomonas aeruginosa, E. coli or non-epidemic antibiotic sensitive Klebsiella of corresponding capsular type. Enhanced survival of multiply-resistant Klebsiella on fingertips may contribute to the transmissibility of these organisms during hospital outbreaks, especially when compared with $E$. coli and Pseudomonas aeruginosa. ${ }^{4}$

Hand contamination can be traced not only to a carrier state but also to accidental contact with an infected patient. Changing of baby diapers led to the acquisition of coliforms on the hands of the nurses in 44 of 49 experiments. 5

Mortimer et al followed the acquisition of staphylococci by infants and nurses from an index infant, known to be a nasal carrier of a certain strain, when the baby was touched or not touched by these nurses. They were able to demonstrate that the transmission of staphylococcal infection occurs mainly by physical transfer on hands. The organisms were carried in the nose, transferred to the hands and from them to the patient. Airborne contamination or the transfer by fomites are unlikely to be common in nurseries and wards but are a potentially important mechanism of transfer in the operating theatre.6

When contaminated objects are moist, about $10 \%$ of the available bacterial cells pass on to the hands and the transfer of organisms from the wet hands to an untouched fabric amounts to $85 \%$. When the hands are dry, only $0.0057 \%$ of the available cells are transferred from a donor fabric to an untouched fabric by contact with the hands. ${ }^{7}$

Transmission by hand contact occurs not only with bacteria but also with more fastidious organisms such as viruses. Not only has hand-to-hand transmission of rhinoviruses been shown, but experimental transfer from the hands to others by an environmental surface has been described. Rotaviruses were detected in handwashings of attendants of children with diarrhea. ${ }^{8-10}$

Rhinoviruses were transmitted from experimental infected volunteers to susceptible recipients: 11 of 15 hand contact exposures initiated infection. Virus on volunteers' hands was transferred to recipients' fingers during $71 \%$ of 10-second hand contact exposures.

Respiratory syncytial virus in freshly obtained infant secretions was recovered from countertops for up to 6 hours, from rubber gloves for up to $1 \frac{1}{2}$ hours, from cloth gowns and paper tissue for 30 to $45 \mathrm{~min}$. and from skin for up to $20 \mathrm{~min}$. Further experiments demonstrated that infectious viruses could be transferred to hands touching these contaminated surfaces and could be recovered from these hands for up to $25 \mathrm{~min} .^{11}$

Nosocomial infections may not only be transmitted by hands, but also from gowns, the nasopharynx, blood and the gastrointestinal tract of hospital staff. Lidwell et al demonstrated that nurses' gowns are often contaminated with strains of Staphylococcus aureus derived from infected patients or carriers and demonstrated that these strains carried on the nurses' external clothing are often transferred to patient bedclothes during bedmaking even in a laminar air flow ventilation system for a single-bed patient room. ${ }^{12}$ This possible route of patient infection was almost eliminated when the nurses wore disposable plastic aprons over their dresses.

Ayliffe and co-workers sampled cotton gowns and plastic aprons over a period of up to 11 days. Staphylococcus aureus was frequently isolated, usually in small numbers from $13 \%$ of cotton gowns, $9.2 \%$ of plastic aprons and $15 \%$ of nurses' uniforms. Gram-negative bacilli were infrequently isolated. Contamination of the protective clothing did not increase when used over a period of up to 11 days. Fewer organisms were recovered from the front of nurses' uniforms when plastic aprons instead of gowns were worn, but gowns provided better shoulder protection. ${ }^{13}$

Nyström compared the bacterial contamination of protective gowns used for 6 and for 24 hours in an intensive care unit with the contamination of protective gowns used in normal wards. There was no significant difference in the mean contamination level between the three groups of gowns studied indicating that a more frequent change to new, clean gowns than every 24 hours in the intensive care unit and twice weekly in the normal ward would be of little practical significance. On occasional gowns in the intensive care unit, very high bacterial counts were observed. ${ }^{14}$

Staphylococcus carried in the nasopharynx of the operating team is one of the main causes of postoperative wound infections. Half of all staphylococcal wound infections are of endogenous origin, the other half of exogenous origin. Most exogenous postoperative staphylococcal wound infections originate not from the air but from the nasopharynx of the surgical team.

Streptococcal wound infections can almost always be traced to a staff carrier. Staff carriers of organisms causing nosocomial gastrointestinal diseases such as $E$. coli, campylobacter, yersinia, or virus infections rarely endanger patients. The risk for the hospital personnel of acquiring these diseases by patients is much greater. The same is true for blood-transmitted diseases such as hepatitis $B$, although large epidemics of hepatitis $B$ infections traced to dentists or oral surgeons have been described.

\section{REFERENCES}

I. Albert RK, Condie F: Hand-washing patterns in medical intensive-care units. N EnglJ Med 1981; 304:1465-1466. 
2. Larson EL: Persistent carriage of gram-negative bacteria on hands. Am J Infect Control 1981; 9:112-119.

3. Adams BG, Marrie TJ: Hand carriage of aerobic gram-negative rods may not be transient. J Hyg 1982; 89:33-46.

4. Casewell MW, Desai N: Survival of multiply-resistant Klebsiella aerogenes and other gram-negative bacilli on finger-tips. J Hosp Infect 1983; 4:350-360.

5. Sprunt K, Redman W, Leidy G: Antibacterial effectiveness of routine hand washing. Pediatrics 1973; 52:264-271.

6. Mortimer EA, Wolinsky E, Gonzaga AJ, et al: Role of airborne transmission in staphylococcal infections. Br Med J 1966; 1:319-322.

7. Reybrouck G: Role of hands in the spread of nosocomial infections. $J$ Hosp Infect 1983; 4:103-110.

8. Samadi AR, Hug MI, Ahmed QS: Detection of rotavirus in handwashings of attendants of children with diarrhea. $\mathrm{Br} \mathrm{Med} J$ 1983; 1:188.

9. Gwaltney $J \mathbf{M}$, Hendley JO: Transmission of experimental rhinovirus infection by contaminated surfaces. Am J Epidemiol 1982; 116:828-833.

10. Gwaltney JM, Moskalski PB, Hendley JO: Hand-to-hand transmission of rhinovirus colds. Ann Intern Med 1978; 88:463-467.

11. Hall CB, Douglas RG, Geiman JM: Possible transmission by fomites of respiratory syncytial virus. I Infect $D$ is 1980; 141:98-102.

12. Lidwell OM, Towers AG, Ballard J, et al: Transfer of microorganisms between nurses and patients in a clean air environment. $J$ Appl Bacteriol 1974; 37:649-656

13. Babb JR, Davies JG, Ayliffe GAJ: Contamination of protective clothing and nurses' uniforms in an isolation ward. J Hosp Infect 1983; 4:149-157.

14. Nyström B: The contamination of gowns in an intensive care unit. J Hosp Infect $1980 ; 2: 167-170$.

Prof. Dr.Med. F. Daschner

Department of Hospital Epidemiology

University Hospital, Freiburg

Freiburg, West Germany 